\title{
Differential miRNA expressions in peripheral blood mononuclear cells for diagnosis of lung cancer
}

\author{
Jie Ma ${ }^{1,2}$, Yanli Lin², Min Zhan³, Dean L Mann², Sanford A Stass² and Feng Jiang ${ }^{2}$
}

Tremendous efforts have been made to develop cancer biomarkers by detecting circulating extracellular miRNAs directly released from tumors. Yet, none of the cell-free biomarkers has been accepted to be used for early detection of non-small cell lung cancer (NSCLC). Peripheral blood mononucleated cells (PBMCs) act as the first line of defense against malignancy in immune system, their dysfunction may occur as an early event in cancer immunogenicity or immune evasion. We proposed to investigate whether analysis of miRNA expressions of PBMCs has diagnostic value for NSCLC. We first used a microarray to analyze PBMCs of 16 stage I NSCLC patients and 16 cancer-free smokers, and identified seven PBMC miRNAs with a significantly altered expression level in NSCLC patients. In a training set of 84 NSCLC patients and 69 cancer-free smokers, a panel of two miRNAs (miRs-19b-3p and -29b-3p) were developed from the seven PBMC miRNAs, producing $72.62 \%$ sensitivity and $82.61 \%$ specificity in identifying NSCLC. Furthermore, the miRNAs could identify squamous cell lung carcinoma (SCC), a major type of NSCLC, with $80.00 \%$ sensitivity and $89.86 \%$ specificity. The expression levels of the miRNAs were independent of disease stage. In a testing set of 56 NSCLC patients and 46 controls, the performance of the biomarkers was reproducibly confirmed. The study presents the first in-depth analysis of PBMC miRNA profile of NSCLC patients. The assessment of PBMC miRNAs may provide a new diagnostic approach for the early detection of NSCLC. Laboratory Investigation (2015) 95, 1197-1206; doi:10.1038/labinvest.2015.88; published online 6 July 2015

Non-small cell lung cancer (NSCLC) is the leading causes of cancer death in the United States and around the world. NSCLC predominantly consists of two major histological types: adenocarcinoma (AC) and squamous cell carcinoma (SCC). Cigarette smoking is the major cause of NSCLC, particularly SCC. NSCLC is usually diagnosed at late stages when the prognosis is poor, resulting in an overall 5-year survival rate of $14 \% .{ }^{1}$ Yet, the parameter for stage I NSCLC patients who receive appropriate treatments is up to $83 \% .{ }^{1}$ Therefore, the early detection of NSCLC in smokers can reduce the mortality of lung cancer. Low-dose computed tomography (LDCT) provides excellent anatomic information and has an increasing role in non-invasive diagnosis of early NSCLC. ${ }^{2}$ However, it has limited ability to differentiate between benign and malignant lesions, particularly centrally located cancers, which are often SCCs. ${ }^{3}$ The development of non-invasive approaches that can be used alone or complement CT for more accurately identifying NSCLC, particularly SCC, is thus clinically important.

During the process of tumor development, cancer cells undergo apoptosis and necrosis and release tumor-associated molecules that can circulate in bloodstream. The circulating extracellular molecules derived from primary, invasive, or metastatic tumors may provide cell-free plasma or serum biomarkers for cancer diagnosis. The level of the circulating extracellular molecules differs tremendously depending on the tumor burden, such as size, stage, and metastasis of the tumors. ${ }^{4}$ Early-stage solid tumors may have higher cell growth potential and lesser frequently undergo apoptosis and necrosis processes compared with advanced stage tumors, thus yielding limited amount of the circulating extracellular molecules. Therefore, the current circulating extracellular biomarkers usually exhibit a low accuracy for the early detection of solid tumors, including NSCLC.

Tumors are recognized by the immune system, and cancer cells can evade the elimination of immunological surveillance by several mechanisms, including developing resistance to $\mathrm{T}$ cell-induced apoptosis or the local expression of immunemodulatory molecules and cytokines. ${ }^{5}$ Furthermore, immune system suppression and evasion might occur as early as a pre-malignant event in the development and progression of cancer. ${ }^{6,7}$ Acting as the immune system's first line of defense

\footnotetext{
${ }^{1}$ Department of Clinical Biochemistry, Jiangsu University School of Medicine, Zhenjiang, Jiangsu Province, China; ${ }^{2}$ Department of Pathology, University of Maryland School of Medicine, Baltimore, MD, USA and ${ }^{3}$ Department of Epidemiology and Public Health, University of Maryland School of Medicine, Baltimore, MD, USA

Correspondence: Dr F Jiang, MD, PhD, Department of Pathology, The University of Maryland School of Medicine, 10 South Pine Street, MSTF 7th floor, Baltimore, MD 21201-1192, USA.

E-mail: fjiang@som.umaryland.edu
}

Received 9 February 2015; revised 3 May 2015; accepted 13 May 2015 
against cancer, peripheral blood mononucleated cells (PBMCs) comprise monocytes, $\mathrm{T}$ cells, $\mathrm{B}$ cells, and natural killer cells. ${ }^{8}$ Differential molecular expression of PBMC may appear as soon as cancer immunogenicity or immune evasion is established, both of which have been shown to occur as early as preneoplastic lesions. ${ }^{9,10}$ Indeed, differential molecular changes of PBMCs have been found in the patients diagnosed with early stage of several types of tumors. ${ }^{8,10,11}$ Importantly, the molecular changes of PBMCs are not reliant on substantial tumor burden, which is a major drawback of the current circulating extracellular biomarkers. ${ }^{10}$ Therefore, molecular profiles of PBMCs may provide new biomarkers for the early detection of malignancies. ${ }^{10}$

miRNAs can suppress the translation of mRNA into protein, and have key roles in the regulation of crucial biological processes. Furthermore, miRNAs have significant impacts on the function of antitumor T cells. ${ }^{12}$ In addition, miRNAs involve in mechanisms of cancer cells in the escaping events in immunological surveillance. ${ }^{12}$ Recently, Wang et $a l^{13}$ evaluated PBMC miRNA expression of healthy individuals and patients with either benign pancreatic diseases or pancreatic cancer, and further found that measuring miR-27a-3p expression level in PBMCs could help discriminate pancreatic cancer from the cancer-free subjects. Therefore, differential PBMC miRNA expression profiles could be of use as cancer biomarkers.

With the objective of developing PBMC miRNA biomarkers for the early detection of NSCLC, we systematically defined PBMC miRNA expression profiles of NSCLC patients. From these profiles, we then identified and validated a panel of PBMC miRNAs that might be used as potential biomarkers for the early-stage NSCLC, particularly SCC.

\section{MATERIALS AND METHODS Patient Cohorts}

The study protocol was approved by the Institutional Review Boards of the University of Maryland Medical Center and the Baltimore VA Medical Center. All subjects were selected and consented when they visited the clinics of the Division of Pulmonary and Critical Care in the two medical centers. Final clinical diagnoses were confirmed with histopathologic examinations of specimens obtained by CT-guided transthoracic needle biopsy, transbronchial biopsy, videotape-assisted thoracoscopic surgery, or surgical resection. The surgical pathologic staging was determined according to the TNM classification of the International Union Against Cancer with the American Joint Committee on Cancer and the International Staging System for Lung Cancer. Histopathologic classification was determined according to the World Health Organization classification. From the recruited cohort, we randomly selected 156 patients diagnosed with NSCLC (49 stage I, 36 II, 37 III, and 34 IV), and 131 cancer-free smokers. All the cancer-free smokers had benign lung diseases and were diagnosed with granulomatous inflammation $(n=72)$, non-specific inflammatory changes $(n=40)$, or lung infections $(n=19)$. None of the individuals had received any cancer therapy before blood collection. Subjects with any prior history of cancer were excluded. All participants with benign lung diseases remained cancer free for a minimum 2-year follow-up. The demographic and clinical information were collected including stage and histological diagnosis, smoking history, and size of pulmonary nodules (PNs). The samples were allocated to three sequential research phases. To define PBMC miRNA expression profiles of NSCLC patients using an array platform, we selected PBMC samples of 16 stage I NSCLC patients and 16 cancer-free controls as an exploratory set. The cases and the controls in this exploratory set were matched 1:1 by age, gender, race, and smoking status as a nested case-control study. The demographic and clinical variables of the exploratory set are shown in Supplementary Table 1. The remaining specimens were divided into in a training set and a testing set. The training set consisted of 84 NSCLC patients with different stages and 69 cancer-free controls. The training cohort was used to validate the defined PBMC miRNA profiles of NSCLC from the exploratory cohort, and then develop a panel of biomarkers for NSCLC. The testing cohort comprised 56 stage I NSCLC and 46 cancer-free subjects for validating the panel of biomarkers. The demographic and clinical variables of the training and testing sets are shown in Tables 1 and 2.

\section{PBMC Collection and Processing}

Peripheral blood was collected in BD Vacutainer spray-coated $\mathrm{K}_{2}$ EDTA Tubes (BD, Franklin Lakes, NJ, USA) as described in our previous reports. ${ }^{14,15}$ Within $2 \mathrm{~h}$ of blood draw, PBMCs were isolated by using Ficoll gradient centrifugation as previously described. ${ }^{10}$ Furthermore, to eliminate the contamination of red blood cell (RBC) miRNAs in the PBMC samples, we suspended the PBMC pellets in PharmLyse RBC lysis solution (BS, San Jose, CA, USA) to completely lyse RBCs as previously described. ${ }^{10}$ PBMCs were transferred into RNAlater (Life Technologies, Grand Island, NY, USA) and then stored at $-80^{\circ} \mathrm{C}$ until use. Total RNA was extracted from the cells using the Qiagen miRNeasy Mini Kit (Qiagen, Valencia, CA, USA) as described in our previous studies. ${ }^{14-16}$ Briefly, cells were lysed in $700 \mu \mathrm{l}$ Qiazol lysis reagent. The lysate was transferred to a new tube with $140 \mu \mathrm{l}$ chloroform and centrifuged at $12000 \mathrm{~g}$ for $15 \mathrm{~min}$ in a $4{ }^{\circ} \mathrm{C}$ microcentrifuge. The upper aqueous phase was transferred to a new microcentrifuge tube and 1.5 volume of $100 \%$ ethanol was added. The contents were mixed and $750 \mu \mathrm{l}$ of the sample was transferred to a Qiagen RNeasy Mini spin column in a collection tube followed by centrifugation at $8000 \mathrm{~g}$ for $15 \mathrm{~s}$. The Qiagen RNeasy Mini spin column was rinsed with $700 \mu \mathrm{l}$ Qiagen RWT buffer and centrifuged at $8000 \mathrm{~g}$ for $15 \mathrm{~s}$. The Qiagen RNeasy Mini spin column was transferred to a new collection tube and centrifuged at $15000 \mathrm{~g}$ for $2 \mathrm{~min}$ at room temperature. Total RNA was eluted by adding $50 \mu \mathrm{l}$ of 
Table 1 The demographic and clinical variables of a training set of non-small cell lung cancer (NSCLC) patients and cancerfree smokers

\begin{tabular}{lccr}
\hline & NSCLC cases $(n=84)$ & Controls $(n=69)$ & $P$-value \\
\hline Age & 64.09 (s.d. 9.26) & 62.39 (s.d. 12.44) & 0.38 \\
Sex & & & 0.36 \\
Female & 29 & 23 & \\
Male & 55 & 46 & 0.09 \\
Race & & 52 & \\
White & 63 & 17 & $<0.001$ \\
African American & 21 & & \\
Pack-years & 46.36 (range, 0-200) & 9.38 (range, 0-88) & \\
Nodule size (cm) & 5.45 (range, 96) & 0.75 (range, 57.73) & $<0.001$ \\
& & & \\
Stage & & & \\
I & 21 & & \\
II & 22 & & \\
III & 21 & & \\
IV & & & \\
Histological type & 40 & & \\
AC & & & \\
SCC & & & \\
\hline
\end{tabular}

AC, adenocarcinoma; SCC, squamous cell carcinoma.
Table 2 The demographic and clinical variables of a testing set of non-small cell lung cancer (NSCLC) patients and cancer-free smokers

\begin{tabular}{|c|c|c|c|}
\hline & NSCLC cases $(n=56)$ & Controls $(n=46)$ & $P$-value \\
\hline Age & 65.12 (s.d. 9.78) & 61.28 (s.d. 11.46) & 0.33 \\
\hline Sex & & & 0.48 \\
\hline Female & 17 & 10 & \\
\hline Male & 39 & 36 & \\
\hline Race & & & 0.45 \\
\hline White & 20 & 16 & \\
\hline African American & 36 & 30 & \\
\hline Pack-years & 48.23 (range, 0-212) & 11.02 (range, 0-89) & $<0.001$ \\
\hline Nodule size (cm) & 5.56 (range, 98) & 0.82 (range, 58.46) & $<0.001$ \\
\hline \multicolumn{4}{|l|}{ Stage } \\
\hline । & 12 & & \\
\hline$\|$ & 14 & & \\
\hline III & 16 & & \\
\hline IV & 14 & & \\
\hline \multicolumn{4}{|l|}{ Histological type } \\
\hline$A C$ & 29 & & \\
\hline SCC & 27 & & \\
\hline
\end{tabular}

$\mathrm{AC}$, adenocarcinoma; SCC, squamous cell carcinoma.

using ExiLENT SYBR Green master mix. Negative controls excluding template from the reverse transcription reaction were performed and profiled like the samples. The amplification was performed in a LightCycler 480 Real-Time PCR System (Roche, San Francisco, CA, USA) in 384-well plates. The amplification curves were analyzed using the Roche LC software, both for the determination of quantification cycle (Cq) (Cq was calculated as the second derivative) and for melting curve analysis.

The amplification efficiency was calculated by using algorithms similar to the LinReg software. All assays were inspected for distinct melting curves and the Tm was checked to be within known specifications for the assay. Furthermore, assays were detected with five Cqs less than the negative control, and with $\mathrm{Cq}<37$ to be included in the data analysis. Data that did not pass these criteria were omitted from any further analysis. NormFinder was used to identify the best normalizer according to the average of assays detected in all samples. All data were normalized to the average of assays detected in all samples (average -assay Cq). In addition, a $t$ test with a cutoff of $P$-value $<0.05$ was used to identify differentially expressed miRNAs between lung cancer patients and controls. 


\section{Quantitative Reverse Transcriptase PCR Analysis of miRNAs}

Quantitative Reverse Transcriptase PCR (qRT-PCR) analysis of miRNAs was performed as described in our previous studies. ${ }^{15-18,20-23}$ Briefly, 100 ng RNA was reversely transcribed by a T100 thermal cycler (Applied Biosystems, Foster City, CA, USA) using miRCURY LNA Universal cDNA Synthesis Kit (Exiqon). The thermocycler parameters were as follows: hold for $60 \mathrm{~min}$ at $42{ }^{\circ} \mathrm{C}$, for $5 \mathrm{~min}$ at $95^{\circ} \mathrm{C}$. For qPCR analysis, after $40 \times$ dilution, $4 \mu \mathrm{l}$ of cDNA was combined with $5 \mu \mathrm{l}$ of miRCURY LNATM Universal RT miRNA PCR ExiLENT SYBR Green master mix (Exiqon) and $1 \mu \mathrm{l}$ PCR primers set to produce a PCR in a total volume of 10.0 $\mu$ l. qPCR was carried out on an CFX96 thermocycler (Bio-Rad, Hercules, CA, USA) at $95^{\circ} \mathrm{C}$ for $10 \mathrm{~min}$, followed by 40 cycles of $95^{\circ} \mathrm{C}$ for $15 \mathrm{~s}$ and $60^{\circ} \mathrm{C}$ for $1 \mathrm{~min}$. qPCR data were analyzed by using the Manager software (Bio-Rad) with an automatic Ct setting for assigning the baseline and threshold for Ct determination. All tests were performed in triplicates. For the normalization of the miRNA qPCR data, we screened the expression of all miRNAs represented in the above array-based panel analysis to find the miRNAs with the minimal variation between the two groups. miR-423-3p was chosen as an internal control, since its expression was very stable in all PBMC samples with no significant difference between the groups determined by normalizer. ${ }^{19}$ Relative expression of a targeted miRNA in a given sample was computed using the equation $2-\Delta \mathrm{Ct}$, where $\Delta \mathrm{Ct}=\mathrm{Ct}$ (targeted miRNA) - Ct (miR-423-3p). Ct values were defined as the fractional cycle number, in which, the fluorescence crossed the fixed threshold. All assays were performed in triplicates. Furthermore, two interplate controls and one notemplate control were carried along in each experiment. The no template control for RT was RNase-free water instead of RNA sample input, and no template control for PCR was RNase-free water instead of RT products input. Furthermore, the release of contaminating miRNAs in PBMCs by hemolysis of blood cells such as RBCs could yield non-specific results in the analysis of PBMC-associated miRNA aberrations. Besides using $\mathrm{RBC}$ lysis solution as described above to maximally reduce the possible contamination, ${ }^{10}$ we analyzed expression levels of RBC-related miRNAs (mir-451 and mir-92a) in all samples using qRT-PCR. The PBMC samples that had expression levels of the hemolysis-related miRNAs were considered to have hemolysis and excluded from the study.

\section{Statistical Analysis}

Sample size consideration: to find PBMC miRNAs that differentially expressed between lung cancer patients and cancer-free controls in the array analysis, we expected the acceptable number of false positives to be 1.0, fold difference between normal and tumor of samples at 2.0, standard deviation of the gene measurements on the base-two logarithmic scale at 0.7 , and desired power at $80 \%$. Given 372 miRNAs included in the real-time PCR panel array, 15 specimens from each group type were required to achieve the statistical criteria. ${ }^{24}$ To develop an optimal miRNA marker panel that can be detected in PBMCs for distinguishing cancer patients from normal controls. We used receiver-operator characteristic (ROC) curve and the area under ROC curve (AUC) to determine sample size. The AUC of H0 (the null hypothesis) was set at 0.5 . H1 represented the alternative hypothesis; accordingly, at least 25 subjects were required in each category to show a minimum difference of interest between an AUC of $0.75 v s$ an AUC of 0.5 with $90 \%$ power at the $5 \%$ significance level. ${ }^{25}$ Therefore, the sample size of 84 NSCLC patients and 69 cancer-free controls in the training set, and 56 NSCLC patients and 46 cancerfree subjects in the training set would provide enough statistical power for the identification and validation of the biomarkers.

Data analysis: statistical analysis of RT-qPCR data was done by using SAA version (SAS Institute, Cary, NC, USA). All $P$-values shown were two sided, and a $P$-value of $<0.05$ was considered as statistically significant. Kruskal-Wallis test for comparing continuous variables or significance test for Spearman's rank correlation coefficient was applied to determine the associations between the expression levels of the miRNAs with both clinicopathologic and demographic characteristics of the cases and the controls. ROC curve analysis was undertaken using expression level for each miRNA in PBMCs from cancer patients and cancer-free controls. For each miRNA, we constructed the ROC curve and computed the AUC value by numerical integration of the ROC curve. Using this approach, the AUC was used to determine the accuracy of a biomarker on its ability to distinguish cases from controls. For each gene, we identified the point on ROC curve that is closest to the perfect point with sensitivity $=1$ and specificity $=1$, yielding corresponding optimal thresholds defining expression levels of the tested genes. A stepwise logistic regression model was used to select the optimal panel of miRNAs.

\section{RESULTS \\ Identifying Differentially Expressed miRNAs in PBMCs of NSCLC Patients}

Of the 372 mature human miRNAs represented on the qPCR-based Exiqon miRNA array, 181 were detectable in all of PBMC samples of 16 NSCLC cases and 16 controls, since they had a $<37 \mathrm{Cq}$ value. Of the 181 miRNAs, seven had $\geq 2.0$ fold-changes $(P$-value $<0.05)$ in NSCLC patients $v s$ the cancer-free smokers (Table 3 ). Of the seven miRNAs, three miRNAs (miRs-576-3p, 19b-3p, and 766-3p) were overexpressed, and four miRNAs (miRs-29b-3p, 101-3p, 29a-3p, and 193b-3p) were underexpressed in PBMCs of NSCLC patients $v s$ cancer-free smokers. Furthermore, altered expressions of the seven miRNAs were observed in $81.3 \%(13 / 16)$ PBMC samples of the NSCLC patients compared with those of the cancer-free controls. Therefore, the seven miRNAs proceed to the next phase of the study. 


\section{Developing a Panel of PBMC miRNA Biomarkers for Diagnosis of NSCLC}

We first confirmed the miRNA results by using qRT-PCR in PBMC samples of a training set of 84 NSCLC patients and 69 cancer-free smokers. All targeted seven miRNAs had $\leq 35 \mathrm{Ct}$ values in each PBMC sample, and therefore were reliably detectable by using a different technique, qRT-PCR. No product was synthesized in the negative control samples. Furthermore, the RBC-related miRNAs (mir-451 and mir92a) displayed $>40 \mathrm{Ct}$ values in all the samples, indicating that the RBC-related miRNAs were not found in the PBMC samples. Therefore, there was no contamination of RBC miRNAs in all the PBMC specimens. Of the seven miRNAs, four (miRs-576-3p, 19b-3p, 29b-3p, and 29a-3p) exhibited significantly different level in PBMC specimens of the NSCLC patients $v s$ cancer-free smokers (all $P<0.05$ ) (Table 4; Figure 1). Furthermore, all the four miRNAs had changes determined by qRT-PCR in the same statistically significant direction as by the Exiqon miRNA array. The validation data created from an independent set of specimens using a

$\begin{aligned} & \text { Table } \mathbf{3} \text { miRNAs displayed } \geq \mathbf{2 . 0} \text { fold-changes }(\boldsymbol{P}<\mathbf{0 . 0 5}) \text { in } \\
& \text { NSCLC } \text { patients } \text { vs cancer-free smokers by using Exiqon miRNA } \\
& \text { array }\end{aligned}$
\begin{tabular}{lcc} 
miRNAs & Fold change (NSCLC patients/controls) & $P$-value \\
\hline hsa-miR-576-3p & 3.844590 & 0.003334 \\
hsa-miR-19b-3p & 3.568544 & 0.017525 \\
hsa-miR-29b-3p & -2.645349 & 0.034577 \\
hsa-miR-766-3p & 2.132458 & 0.026767 \\
hsa-miR-101-3p & -2.218565 & 0.036754 \\
hsa-miR-29a-3p & -2.347886 & 0.023568 \\
hsa-miR-193b-3p & -2.570955 & 0.015466 \\
\end{tabular}

NSCLC, non-small cell lung cancer. different approach imply that the four miRNAs could be PBMC signatures of NSCLC.

We further evaluated the potential of using the four PBMC miRNAs for diagnosis of NSCLC in this set of 84 cases and 69 controls. As shown in Table 4, the four PBMC miRNAs exhibited AUC values of $0.61-0.76$ in distinguishing NSCLC patients from cancer-free smokers. A stepwise logistic regression model was used to select the optimal panel of miRNAs. Two miRNAs, miRs-19b-3p and 29b-3p, were selected as the best biomarkers in a panel. The optimal cutoff for the combined biomarkers was $U=0.59898$, where $U=-7.37883$ $+4.08104^{\star} \log (\mathrm{miR}-19 \mathrm{~b}-3 \mathrm{p})-2.8917^{\star} \log (\mathrm{miR}-29 \mathrm{~b}-3 \mathrm{p})$. Any subject with $U \geq 0.59898$ was classified as an NSCLC case. The combined use of the two PBMC miRNAs produced 0.81 AUC (Figure 2a) in distinguishing NSCLC patients from the controls, which was significantly higher than that of any single one of the four miRNAs (Supplementary Table 2) $(P<0.05)$. Furthermore, combined analysis of all four miRNAs did not display a higher AUC value compared with this two miRNAbased biomarker panel (Supplementary Table 2) $(P>0.05)$. In addition, including any additional miRNA in the small panel of two miRNAs did not show a greater AUC compared with the panel of two miRNAs used alone (Supplementary Table 2) $(P>0.05)$. As a result, the analysis of the two miRNAs in PBMC samples produced $72.62 \%$ sensitivity and $82.61 \%$ specificity for diagnosis of NSCLC. Interestingly, the two miRNAs used in combination created a higher AUC value (AUC $=0.90,95 \%$ CI, 0.85-0.97) in distinguishing SCC cases compared with AC cases (AUC $=0.76,95 \% \mathrm{CI}, 0.65-0.87$ ) from controls $(P=0.03)$ (Figure $2 \mathrm{~b}$ and $\mathrm{c})$. Subsequently, the panel of the two miRNA biomarkers had $80.00 \%$ sensitivity and $89.86 \%$ specificity for SCC, and $75.00 \%$ sensitivity and $68.12 \%$ specificity for AC. Therefore, the biomarker panel could better differentiate SCC cases from controls compared with AC cases. Moreover, the two PBMC miRNAs did not display statistical differences of expression between stages of NSCLC ( $P=0.18$ and 0.85 , respectively) (Supplementary

Table 4 Expression levels of the miRNAs in PBMC samples of NSCLC patients vs cancer-free smokers by using qRT-PCR assay

\begin{tabular}{lcccc}
\hline MiRNA & $\begin{array}{c}\text { Mean } \pm \text { s.e.m. in } \\
\text { cancer-free controls }\end{array}$ & $\begin{array}{c}\text { Mean } \pm \text { s.e.m. in } \\
\text { NSCLC patients }\end{array}$ & P-value & AUC \\
\hline miR-576-3p & $0.0045 \pm 0.0002$ & $0.0060 \pm 0.00023$ & $<\mathbf{0 . 0 0 0 1}$ & $\mathbf{0 . 7 5 7 6}$ (Std. Error, 0.0464; 95\% confidence interval, 0.6667-0.8486) \\
miR-19b-3p & $24.25 \pm 0.6373$ & $30.09 \pm 0.8643$ & $<\mathbf{0 . 0 0 0 1}$ & $\mathbf{0 . 7 5 4 6}$ (Std. Error, 0.0457; 95\% confidence interval, 0.6651-0.8441) \\
miR-29b-3p & $8.134 \pm 0.2778$ & $6.541 \pm 0.1923$ & $<\mathbf{0 . 0 0 0 1}$ & $\mathbf{0 . 7 5 3 6}$ (Std. Error, 0.0483; 95\% confidence interval, 0.6589-0.8482) \\
miR-29a-3p & $16.31 \pm 0.6789$ & $14.65 \pm 0.4243$ & $\mathbf{0 . 0 3 2 5}$ & $\mathbf{0 . 6 0 5 0}$ (Std. Error, 0.0551; 95\% confidence interval, 0.4969-0.7131) \\
miR-766-3p & $0.2802 \pm 0.0144$ & $0.2741 \pm 0.0138$ & $\mathbf{0 . 7 6 7 7}$ & $\mathbf{0 . 5 2 9 6}$ (Std. Error, 0.0564; 95\% confidence interval, 0.4190-0.6401) \\
miR-101-3p & $5.412 \pm 0.1799$ & $5.805 \pm 0.1755$ & $\mathbf{0 . 1 2 9 1}$ & $\mathbf{0 . 6 0 6 0}$ (Std. Error, 0.0553; 95\% confidence interval, 0.4976-0.7143) \\
miR-193b-3p & $0.0661 \pm 0.0079$ & $0.0627 \pm 0.0038$ & $\mathbf{0 . 6 7 8 6}$ & $\mathbf{0 . 5 3 1 6}$ (Std. Error, 0.0556; 95\% confidence interval, 0.4226-0.6406) \\
\hline
\end{tabular}

AUC, the area under receiver-operator characteristic curve; NSCLC, non-small cell lung cancer; PBMC, peripheral blood mononucleated cells; qRT-PCR, quantitative reverse transcriptase PCR; s.e.m., standard error of the mean. The AUC values that have $P$-values less than 0.05 are indicated in bold characters. 

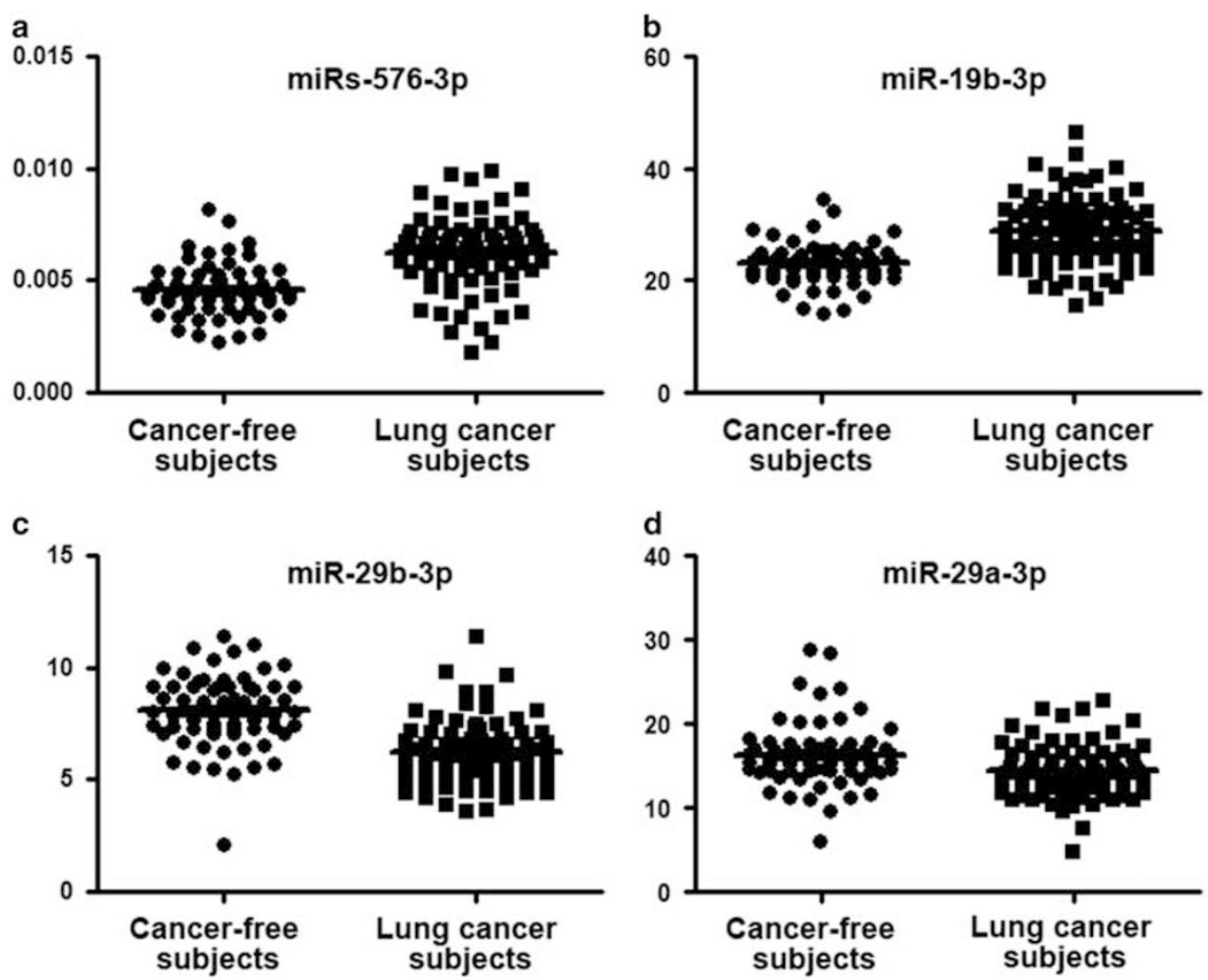

Figure 1 Expression levels of the four miRNAs ((a) miRs-576-3p, (b) miR-19b-3p, (c) miR-29b-3p, and (d) miR-29a-3p)) in PBMC samples of 46 cancer-free smokers and 64 NSCLC patients. The inside line denotes the median. All the four PBMC miRNAs have statistically significantly different levels in the NSCLC patients vs cancer-free smokers (all $P<0.05$ ).

Table 3). The panel of two biomarkers did not exhibit significant association with age and ethnicity of the participants (all $P \geq 0.05$ ) (Supplementary Table 3). The expression level of the PBMC miR-29b-3p was correlated with size of PNs $(P=0.01)$. The expression levels of both miR-19b-3p and miR-29b-3p were associated with smoking history of subjects $(P=0.02)$.

\section{Validating the Panel of PBMC miRNA Biomarkers in a Testing Cohort of Specimens}

The diagnostic value of the miRNA panel was validated in a testing cohort in a blinded manner using the optimal threshold established in the training set. Overall, the combined use of the two PBMC miRNAs produced $71.43 \%$ sensitivity and $82.61 \%$ specificity in distinguishing NSCLC cases from controls. Furthermore, the small panel of the biomarkers had $77.78 \%$ sensitivity and $89.13 \%$ specificity for SCC, whereas $75.86 \%$ sensitivity and $67.39 \%$ specificity for AC. The results generated from a different cohort of specimens confirmed the potential of the PBMC miRNAs as potential biomarkers for the early detection of NSCLC, particularly SCC.

\section{DISCUSSION}

Our present study may represent the first in-depth analysis of PBMC miRNA profile of NSCLC patients using a microarray platform. By following the paradigm for biomarker development established by the National Cancer Institute Early Detection Research Network, we validate the PBMC miRNA profile and then develop a panel of two biomarkers (miRs-19b-3p and 29b-3p) for NSCLC in a training cohort of cases and controls. The panel of the PBMC miRNA biomarkers can discriminate lung cancer patients from control subjects with $72.62 \%$ sensitivity and $82.61 \%$ specificity. Interestingly, the biomarker panel could diagnose SCC with $80.00 \%$ sensitivity and $89.86 \%$ specificity. The diagnostic performance of the biomarkers was further validated in a testing set of specimens. Moreover, the sensitivity and specificity of the biomarkers are independent of disease stage. Therefore, the discovery might be an important characteristic if they are employed for the diagnosis of NSCLC, particularly SCC, at the early stage.

Previous reports including our own data have shown that numerous miRNAs display abnormal expressions in NSCLC tissues compared with normal lung tissues. ${ }^{16,26-29}$ Of the seven dysregulated PBMC miRNAs of NSCLC patients profiled by the array analysis, only one (miR-19b-3p) was previously found to be differentially expressed in primary NSCLC tissues, suggesting that the PBMC miRNA profile of NSCLC might have little similarity with those in lung cancer tissues. The observation implies that the miRNA expression 

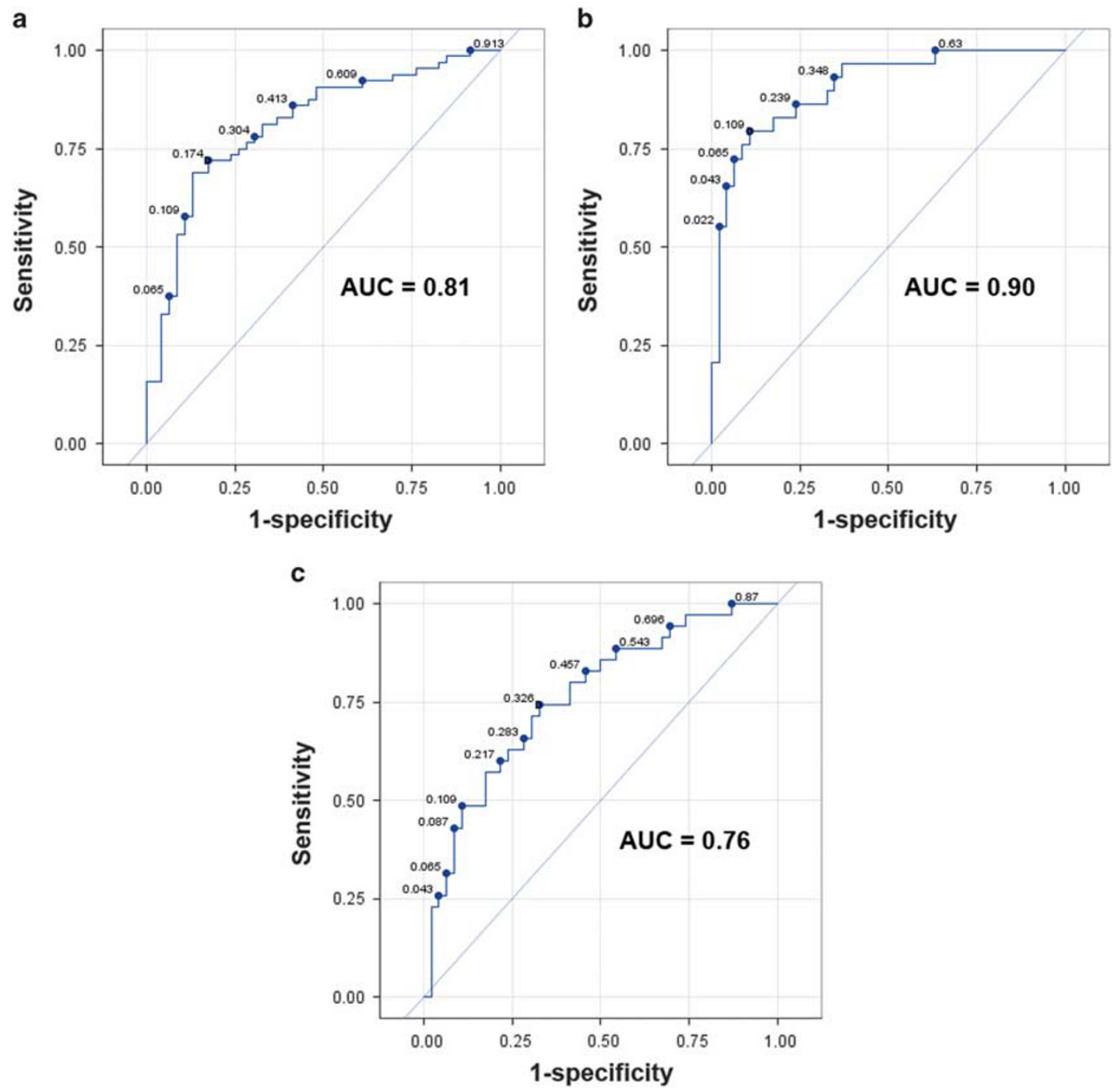

Figure 2 Receiver-operator characteristic (ROC) curve analysis of two PBMC miRNAs (miRs-19b-3p and-29b-3p) in a training set of NSCLC patients and cancer-free smokers. The area under the ROC curve (AUC) for each miRNA conveys its accuracy for diagnosis of NSCLC. The panel of the two PBMC miRNA biomarkers produces a higher AUC value for identifying SCC (b), compared with NSCLC (a) and AC (c), respectively (all $P<0.05)$.

profile in PBMCs may not be a reflection of the miRNA expressed in the NSCLC tissues, or mimic that in the primary tumors. Furthermore, previous reports have shown that the assessment of circulating extracellular plasma or serum miRNAs derived from primary tumors could potentially diagnose lung cancer. For instance, we have identified a panel of plasma miRs-21, 210, and 486-5p that had $75.00 \%$ sensitivity and $84.95 \%$ specificity in distinguishing lung cancer patients from subject with benign diseases. ${ }^{15}$ However, there is no overlap between the PBMC miRNA profile of NSCLC $v s$ the circulating extracellular plasma or serum miRNAs from lung tumors. ${ }^{15,30,31}$ Therefore, miRNA expression in PBMCs is not likely to be influenced by circulating tumor cells. In addition, PBMC miRNA profiles have also been identified to associate with other cancer types. For examples, Wang et $a l^{13}$ found that the assessment of
miR-27a-3p level in PBMCs could discriminate pancreatic cancer from healthy subjects and patients with benign pancreatic/peripancreatic diseases. There is no similarity of the differentially expressed PBMC miRNAs between NSCLC vs other tumors. Taken together, the differential PBMC miRNA expression might be specific to NSCLC, and thus support the potential of using the miRNA expression profiling of PBMCs as a diagnostic tool for NSCLC.

Tremendous efforts have been made to develop biomarkers by detecting circulating cell-free miRNAs that escape from malignant cells and end up as detectable ejecta in circulation as a result of apoptotic and necrotic cell death, or active release. ${ }^{25,28,31-36}$ Although showing promise for cancer early detection, the examination of the circulating extracellular miRNAs has some pitfalls that limit its application in the clinical settings. These particularly include (1) low recovery of 
miRNAs in plasma or serum. ${ }^{37}$ (2) The release of miRNAs in plasma or serum by hemolysis of blood cells such as RBCs, producing non-specific results. (3)Sources of variability of circulating extracellular plasma miRNAs may lead to inconsistent or even contradictory results for the diagnosis of the same type of cancer between reports. ${ }^{38}$ The problem becomes more complicated by the fact that in blood, miRNAs are either associated with proteins, such as argonaute, ${ }^{38,39}$ lipoproteins, ${ }^{40}$ or contained within cellular fragments designated as exosomes, microparticles, microvesicles, or extracellular vesicles. ${ }^{41,42}$ Furthermore, from 2008 to 2013, a total of 154 circulating extracellular miRNA expression profiles were identified in 26 different tumor types. ${ }^{31}$ However, no single diagnostic signature emerges between the various reports. ${ }^{31}$ Particularly, a very weak degree of correlation of the miRNA expression profiles is observed for diagnostic signatures in NSCLC. ${ }^{31}$ Given the lack of concordance between most miRNA signatures in a given tumor type and the mixed correlations between circulating and tissue-specific levels of these reported miRNAs, there is an urgent need for a new approach that could help develop miRNAs as reliable non-invasive biomarkers. ${ }^{31}$

The analysis of PBMC miRNA biomarkers may overcome the obstacles, since it is a cell-based and easily performed approach. Furthermore, PBMCs can yield large amounts of RNA with high quality for reliable determination of miRNAs by using qRT-PCR. In addition, RNA is specifically isolated from the purified PBMCs rather than cell-free or circulating plasma, and thus may not contain RBC miRNAs. Indeed, our examination of the hemolysis-related RBC miRNAs in PBMCs by using specific primers and probes indicates that there is no erythrocyte miRNA contamination. Moreover, compared with the circulating extracellular biomarkers that are reliant on significant tumor burden, PBMC miRNA biomarkers might theoretically have higher diagnostic value, because the altered PBMC miRNA expression is involved in immune system's recognition of cancer and evasion of the immune system by tumor cells. ${ }^{10}$ The immunological recognition of cancer and surveillance occur as early events in tumorigenesis. ${ }^{10}$ Therefore, the determination of differential PMBC miRNA expression may act as a surrogate window into cancer status. ${ }^{10}$ However, conducting a new study to simultaneously analyze and directly compare miRNA profiles of paired PBMCs, plasma, and tumor tissues of the NSCLC patients will determine whether the PBMC miRNAs are independent from those in blood and tumors of NSCLC patients, which have a better diagnostic value, and if they are complementary to each other in the early detection of NSCLC.

Showe $e a^{43}$ previously analyzed gene expression at mRNA level in PBMCs of 137 patients with NSCLC tumors and 91 patient controls with non-malignant diseases, and identified a 29-gene signature that separates these two patient classes with $91 \%$ sensitivity and $80 \%$ specificity. The sensitivity of the gene signature was reduced when validated in an independent validation set (76 vs 91\% sensitivity). Although consisting of only two PBMC miRNAs, our developed biomarker panel produced similar specificity ( 83 vs $80 \%$ ) and a slight lower sensitivity (73 vs 76\%) for diagnosis of NSCLC compared with the 29-gene signature. Furthermore, the performance of this small panel of PBMC miRNA biomarkers was reproducibly validated in a different set of cases and controls. Importantly, the panel of the PBMC miRNA biomarkers could identify SCC with a much higher specificity ( 89 vs 80\%) compared with the gene signature. Given that LDCT has limited capability to differentiate between benign and malignant lesions, particularly centrally located SCCs, ${ }^{2,3}$ future use of the PBMC miRNA biomarkers would help make decisions about the management of CT-detected abnormalities of undetermined importance. In addition, due to the smaller size, more stability, and resistance to RNase activity, miRNAs might be better suited for profiling than mRNAs and large intergenic non-coding RNAs that are easily subject to degradation in clinical samples. ${ }^{31,42}$ However, identifying additional PBMC miRNA biomarkers that can be added to the small panel of biomarkers, and integrating the gene signatures with the PBMC miRNA biomarkers to improve the non-invasive biomarkers for NSCLC is required.

Beyond the diagnostic potential, the identified PBMC miRNAs might have important function in the evasion of immunological surveillance, and thus contribute to lung tumorigenesis. Indeed, miR-29 family express in both $\mathrm{T}$ and $\mathrm{B}$ cells, and the main accessory cell types of thymic epithelium and dendritic cells. ${ }^{44}$ Furthermore, the miR-29 family has the critical role in setting the molecular threshold for essential events in adaptive immunity. ${ }^{44}$ Interestingly, of the ten pathways with the most significant enrichment for predicted targets of miR-29, five have a critical role in the adaptive immune system. ${ }^{44}$ In addition, miR-29b inhibits IFN-r production in primary $\mathrm{CD} 4+$ and $\mathrm{CD} 8+\mathrm{T}$ cells by directly targeting IFN-r mRNA. ${ }^{45}$ miR-29 is upregulated in aggressive B-cell chronic lymphocytic leukemia (B-CLL), and further upregulated in indolent B-CLL, indicating an oncogenic function for miR-29 in B cells. miR-29 downregulation is a poor prognostic marker in aggressive B-CLL. Furthermore, miR-29 can downregulate function of Tcl1, an important oncogene in aggressive B-CLL and a direct target of miR-29, implying a tumor suppressor function of miR-29. ${ }^{44}$ Therefore, the miR-29 family may have complex nature in B-cell oncogenesis. Interestingly, dysregulation of miRs-19b-3p has not been previously reported in the regulation of immune system. Further study is required to investigate the mechanisms, by which the miRNAs involve in cancer cells' evasion of immunological surveillance as an early event in tumorigeneis of NSCLC, particularly SCC.

Five (miRs-576-3p, 766-3p, 29a-3p, 101-3p, and 193b-3p) of the seven PBMC miRNAs whose expressions are associated with lung cancer are not included in the small panel of biomarkers. However, previous studies have suggested that the five miRNAs might also have important function 
in the progression and development of various malignancies. For example, the overexpression of miR-576-3p inhibited bladder cancer cell proliferation via G1-phase arrest, and decreased protein levels of cyclin D1 through binding its $3^{\prime}$-UTR sequences. ${ }^{46}$ Single nucleotide variations of miR-766-3p were identified in lung adenocarcinoma tumors and the changes might have the potential to alter the expression of TNFRSF19. ${ }^{47}$ miR-29a-3p had a low expression level in gastric tumor tissues and its enforced expression inhibited cancer cell proliferation by reducing the expression of CDK2, CDK4, and CDK6. ${ }^{48} \mathrm{miR}-101$ can suppress cell proliferation, invasiveness, and self-renewal in aggressive endometrial cancer cells via modulating multiple critical oncogenes, including EZH2, MCL-1, and FOS. ${ }^{49}$ miR-193b$3 p$ could negatively target KRAS, TYMS, ABCC3, CBL- $B$, and $E R B B 2$ by a post-transcriptional mechanism, and was one of the miRNAs whose dysregulation might have an impact on chemotherapy resistance in esophageal cancer. ${ }^{50}$

Although our results are promising, there are several limitations in the present study. (1) We use a qPCR-based array that only target 372 miRNAs to define differentially expressed PBMC miRNAs of NSCLC patients. Of the 372 miRNAs, only 181 (less than half) could reliably be detectable in the tested samples. Other important lung cancer-associated PBMC miRNAs may not be included in the platform or steadfastly measurable by using the technique. Furthermore, from the measurable 181 miRNAs, only two biomarkers are selected. Therefore, the diagnostic efficiency is still not sufficient to be used in clinical settings. Identifying new PBMC miRNA signatures of NSCLC by using a comprehensive tool (e.g., whole genome next-generation sequencing) might provide additional biomarkers to improve the efficiency of the PBMC miRNA-based assay for lung cancer. (2) The PBMC samples used in this study are obtained from the hospital-based patients with clinical diagnosis. The individuals are not representative of the heavy smokers in screening setting for lung cancer early detection. Plans for the validation of the biomarkers in a prospective and multisite lung cancer screening trial are in progress. (3) To refine the PBMC miRNAs whose changes are specific to lung cancer, we include NSCLC cases and cancer-free smokers who have inflammatory diseases in this study. Using the cohorts consisting of NSCLC cases and cancer-free patients with benign diseases may allow removing confounding effects of the benign diseases on PBMC miRNAs changes, and help identify specific biomarkers for NSCLC. However, the packyears of the NSCLC patients were significantly higher than those of the controls in both training and testing sets (Tables 1 and 2). Recruiting control subjects who have similar pack-years compared with cancer patients and analyzing the PMBC miRNAs in the cases and controls will be necessary to determine whether the differential miRNA expressions were specific to NSCLC rather than smoking. Furthermore, it would be interesting to evaluate whether there is any different expression of the PBMC miRNAs between smokers with benign diseases and healthy non-smokers.

In summary, we have shown that a differential miRNA expression profile exists in PBMCs of NSCLC patients. Further, a panel of PBMC miRNA biomarkers has been established that may have diagnostic potential to improve the early detection of NSCLC, particularly SCC. Given that differential molecular changes of PBMCs can occur as an early event in tumorigenesis, ${ }^{10}$ the PBMC miRNA biomarkers might be developed as a screening tool for lung cancer in high-risk patients in future. Nonetheless, performing an external validation study of the PBMC miRNA biomarkers for lung cancer in specimens prospectively collected from different medical centers is required.

Supplementary Information accompanies the paper on the Laboratory
Investigation website (http://www.laboratoryinvestigation.org)

\section{ACKNOWLEDGMENTS}

This work was supported in part by NCI R01CA161837, VA merit Award 101 CX000512, LUNGevity/Upstage Foundation Early Detection Award, and University of Maryland Cancer Epidemiology Alliance Seed Grant (FJ), and 5U24CA11509 (SS), and award from the Geaton and JoAnn DeCesaris Family Foundation (FJ).

\section{DISCLOSURE/CONFLICT OF INTEREST}

The authors declare no conflict of interest.

1. Cancer Facts \& Figures 2012. American Cancer Society (ACS). J Consum Health Internet 2012;16:366-367.

2. Aberle DR, Adams AM, Berg CD et al. Reduced lung-cancer mortality with low-dose computed tomographic screening. N Engl J Med 2011;365:395-409.

3. Toloza EM, Harpole L, McCrory DC. Noninvasive staging of non-small cell lung cancer: a review of the current evidence. Chest 2003;123: 137S-146SS.

4. Ilie $M$, Hofman V, Long $E$ et al. Current challenges for detection of circulating tumor cells and cell-free circulating nucleic acids, and their characterization in non-small cell lung carcinoma patients. What is the best blood substrate for personalized medicine? Ann Transl Med 2014;2:107.

5. Melief CJ, Finn OJ. Cancer immunology. Curr Opin Immunol 2011;23: 234-236.

6. Keenan B, Jaffee EM. Immunotherapy in preneoplastic disease: targeting early procarcinogenic inflammatory changes that lead to immune suppression and tumor tolerance. Ann NY Acad Sci 2013;1284:12-16.

7. Inman KS, Francis AA, Murray NR. Complex role for the immune system in initiation and progression of pancreatic cancer. World J Gastroenterol 2014;20:11160-11181.

8. Twine NC, Stover JA, Marshall B et al. Disease-associated expression profiles in peripheral blood mononuclear cells from patients with advanced renal cell carcinoma. Cancer Res 2003;63:6069-6075.

9. Bluth $\mathrm{M}$, Lin $\mathrm{YY}$, Zhang $\mathrm{H}$ et al. Use of gene expression profiles in cells of peripheral blood to identify new molecular markers of acute pancreatitis. Arch Surg 2008;143:227-233.

10. Baine MJ, Chakraborty S, Smith LM et al. Transcriptional profiling of peripheral blood mononuclear cells in pancreatic cancer patients identifies novel genes with potential diagnostic utility. PLoS One 2011;6:e17014.

11. Whitney AR, Diehn M, Popper SJ et al. Individuality and variation in gene expression patterns in human blood. Proc Natl Acad Sci USA 2003;100:1896-1901.

12. Sasaki K, Kohanbash G, Hoji A et al. miR-17-92 expression in differentiated T cells-implications for cancer immunotherapy. J Transl Med 2010;8:17. 
13. Wang WS, Liu LX, Li GP et al. Combined serum CA19-9 and miR-27a-3p in peripheral blood mononuclear cells to diagnose pancreatic cancer. Cancer Prev Res (Phila) 2013;6:331-338.

14. Ma J, Li N, Guarnera M, Jiang F. Quantification of plasma miRNAs by digital PCR for cancer diagnosis. Biomark Insights 2013;8:127-136.

15. Shen J, Liu Z, Todd NW et al. Diagnosis of lung cancer in individuals with solitary pulmonary nodules by plasma microRNA biomarkers. BMC Cancer 2011;11:374.

16. Shen J, Todd NW, Zhang $\mathrm{H}$ et al. Plasma microRNAs as potential biomarkers for non-small-cell lung cancer. Lab Invest 2011;91:579-587.

17. Xing L, Su J, Guarnera MA et al. Sputum microRNA biomarkers for identifying lung cancer in indeterminate solitary pulmonary nodules. Clin Cancer Res 2015;21:484-489.

18. Shen J, Liao J, Guarnera MA et al. Analysis of MicroRNAs in sputum to improve computed tomography for lung cancer diagnosis. J Thorac Oncol 2014:9:33-40.

19. Ralfkiaer U, Hagedorn PH, Bangsgaard $\mathrm{N}$ et al. Diagnostic microRNA profiling in cutaneous T-cell lymphoma (CTCL). Blood 2011;118. 5891-5900.

20. Ma J, Mannoor K, Gao L et al. Characterization of microRNA transcriptome in lung cancer by next-generation deep sequencing. Mol Oncol 2014;8:1208-1219.

21. Yu L, Todd NW, Xing $L$ et al. Early detection of lung adenocarcinoma in sputum by a panel of microRNA markers. Int J Cancer 2010;127: 2870-2878.

22. Xing L, Todd NW, Yu L et al. Early detection of squamous cell lung cancer in sputum by a panel of microRNA markers. Mod Pathol 2010;23:1157-1164

23. Wang J, Tian X, Han R et al. Downregulation of miR-486-5p contributes to tumor progression and metastasis by targeting protumorigenic ARHGAP5 in lung cancer. Oncogene 2014;33:1181-1189.

24. Sahai $H$, Khurshid $A$. Formulae and tables for the determination of sample sizes and power in clinical trials for testing differences in proportions for the two-sample design: a review. Stat Med 1996;15: $1-21$.

25. Obuchowski NA, Lieber ML, Wians Jr FH. ROC curves in clinical chemistry: uses, misuses, and possible solutions. Clin Chem 2004;50: 1118-1125.

26. Shen J, Jiang F. Applications of MicroRNAs in the diagnosis and prognosis of lung cancer. Expert Opin Med Diagn 2012;6:197-207.

27. Boeri M, Verri C, Conte D et al. MicroRNA signatures in tissues and plasma predict development and prognosis of computed tomography detected lung cancer. Proc Natl Acad Sci USA 2011;108: 3713-3718.

28. Yanaihara N, Caplen N, Bowman E et al. Unique microRNA molecular profiles in lung cancer diagnosis and prognosis. Cancer Cell 2006;9: 189-198.

29. Hu Z, Chen $X$, Zhao $Y$ et al. Serum microRNA signatures identified in genome-wide serum microRNA expression profiling predict survival of non-small-cell lung cancer. J Clin Oncol 2010;28:1721-1726.

30. Sozzi G, Boeri M, Rossi M et al. Clinical utility of a plasma-based miRNA signature classifier within computed tomography lung cancer screening: a correlative MILD trial study. J Clin Oncol 2014;32:768-773.

31. Jarry J, Schadendorf D, Greenwood C et al. The validity of circulating microRNAs in oncology: five years of challenges and contradictions. Mol Oncol 2014;8:819-829.
32. Cortez MA, Bueso-Ramos C, Ferdin J et al. MicroRNAs in body fluidsthe mix of hormones and biomarkers. Nat Rev Clin Oncol 2011;8: 467-477.

33. Valadi $\mathrm{H}$, Ekstrom $\mathrm{K}$, Bossios $\mathrm{A}$ et al. Exosome-mediated transfer of mRNAs and microRNAs is a novel mechanism of genetic exchange between cells. Nat Cell Biol 2007;9:654-659.

34. Pegtel DM, Cosmopoulos K, Thorley-Lawson DA et al. Functional delivery of viral miRNAs via exosomes. Proc Natl Acad Sci USA 2010;107:6328-6333.

35. Wang K, Zhang S, Weber J et al. Export of microRNAs and microRNAprotective protein by mammalian cells. Nucleic Acids Res 2010;38: 7248-7259.

36. Vickers KC, Remaley AT. Lipid-based carriers of microRNAs and intercellular communication. Curr Opin Lipidol 2012;23:91-97.

37. Fichtlscherer S, De Rosa S, Fox $\mathrm{H}$ et al. Circulating microRNAs in patients with coronary artery disease. Circ Res 2010;107:677-684.

38. Moldovan L, Batte KE, Trgovcich J et al. Methodological challenges in utilizing miRNAs as circulating biomarkers. J Cell Mol Med 2014;18: 371-390.

39. Arroyo JD, Chevillet JR, Kroh EM et al. Argonaute2 complexes carry a population of circulating microRNAs independent of vesicles in human plasma. Proc Natl Acad Sci USA 2011;108:5003-5008.

40. Vickers KC, Palmisano BT, Shoucri BM et al. MicroRNAs are transported in plasma and delivered to recipient cells by high-density lipoproteins. Nat Cell Biol 2011;13:423-433.

41. Hunter MP, Ismail N, Zhang $X$ et al. Detection of microRNA expression in human peripheral blood microvesicles. PLoS One 2008;3:e3694.

42. Mitchell PS, Parkin RK, Kroh EM et al. Circulating microRNAs as stable blood-based markers for cancer detection. Proc Natl Acad Sci USA 2008;105:10513-10518.

43. Showe MK, Vachani A, Kossenkov AV et al. Gene expression profiles in peripheral blood mononuclear cells can distinguish patients with nonsmall cell lung cancer from patients with nonmalignant lung disease. Cancer Res 2009;69:9202-9210.

44. Liston A, Papadopoulou AS, Danso-Abeam D et al. MicroRNA-29 in the adaptive immune system: setting the threshold. Cell Mol Life Sc 2012;69:3533-3541.

45. Ma F, Xu S, Liu X et al. The microRNA miR-29 controls innate and adaptive immune responses to intracellular bacterial infection by targeting interferon-gamma. Nat Immunol 2011;12:861-869.

46. Liang Z, Li S, Xu X et al. MicroRNA-576-3p inhibits proliferation in bladder cancer cells by targeting cyclin D1. Mol Cells 2015;38:130-137.

47. Sabarinathan R, Wenzel A, Novotny $P$ et al. Transcriptome-wide analysis of UTRs in non-small cell lung cancer reveals cancer-related genes with SNV-induced changes on RNA secondary structure and miRNA target sites. PLoS One 2014;9:e82699.

48. Zhao Z, Wang L, Song $W$ et al. Reduced miR-29a-3p expression is linked to the cell proliferation and cell migration in gastric cancer. World J Surg Oncol 2015;13:101.

49. Konno $\mathrm{Y}$, Dong $\mathrm{P}$, Xiong $\mathrm{Y}$ et al. MicroRNA-101 targets EZH2, MCL-1 and FOS to suppress proliferation, invasion and stem cell-like phenotype of aggressive endometrial cancer cells. Oncotarget 2014;5: 6049-6062.

50. Hummel R, Sie C, Watson DI et al. MicroRNA signatures in chemotherapy resistant esophageal cancer cell lines. World J Gastroenterol 2014;20:14904-14912. 